\title{
Gaseous Haloes: Linking Galaxies to the IGM
}

\author{
Filippo Fraternali
}

Department of Astronomy, University of Bologna, Italy

email: filippo.fraternali@bo.astro.it

\begin{abstract}
Recent observations show that spiral galaxies are surrounded by massive and extended haloes of both neutral and ionized gas. These gaseous haloes have peculiar kinematics with respect to their discs: they rotate more slowly and they show an overall inflow motion. Theoretical models of the formation of these gaseous haloes show that they cannot originate solely from a galactic fountain (supernova outflows from the disk); substantial accretion of material from the Intergalactic Medium should play an important role.
\end{abstract}

\section{Gaseous Haloes}

Recent deep neutral hydrogen (HI) observations of several nearby spiral galaxies indicate that a large fraction of the neutral gas in these objects is not located in the disc but in the halo region (e.g. NGC 891, Swaters, Sancisi, \& van der Hulst (1997); M31, Westmeier, Braun, \& Thilker (2005); UGC 7321, Matthews \& Wood (2003)). The halo gas rotates more slowly than the gas in discs (e.g. Fraternali et al. (2002)), shows strong vertical motions (Boomsma et al. (2005)) and an overall inflow motion (e.g. Fraternali et al. (2001)). Gaseous haloes are also detected in optical emission line (e.g. Hoopes, Walterbos \& Rand (1999), Rossa \& Dettmar (2003)) with kinematics similar to that of the neutral gas (Heald et al. (2006b)) and in X-rays (e.g. Strickland et al. (2004)). The gaseous haloes around external galaxies are analogous to the Intermediate and High Velocity Clouds (IVCs and HVCs) of the Milky Way (Wakker \& van Woerden (1997)).

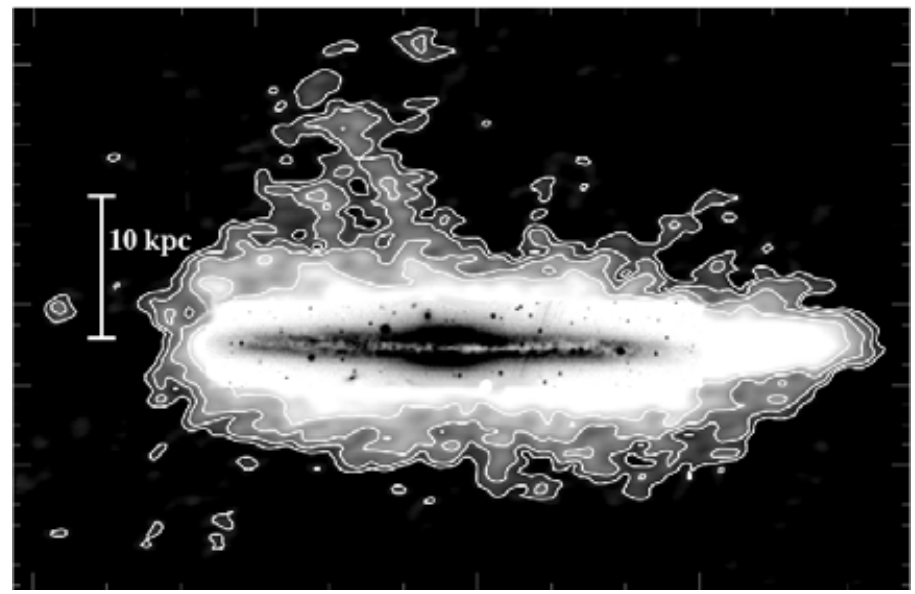

Figure 1. Optical DSS image (grey-scale) and total H I map (contours+negative grey-scale) of NGC 891. The H I contours are $1,2,4,8,16 \times 10^{19}$ atoms $\mathrm{cm}^{-2}$. The beam size is $25^{\prime \prime}=$ $1.15 \mathrm{kpc}$. 
New deep (200 hrs integration) H I observations of the nearby edge-on spiral galaxy NGC 891 obtained with the Westerbork Synthesis Radio Telescope show an extended and massive ( $\sim 30 \%$ of the total H I mass) halo of neutral gas (Oosterloo, Fraternali \& Sancisi (2006)). Fig. 1 shows the total H I map of NGC 891 (contours + white shade) with overlaid an optical image (grey-scale). The map is rotated 67 degrees counter-clock wise with respect to the on-sky orientation. The neutral gas extends to large heights $(z \gtrsim 8 \mathrm{kpc})$ from the plane at all radii. Some features, in particular the long filament visible in the upper left quadrant, extend to larger distances $(\sim 20 \mathrm{kpc})$. The kinematics of the halo gas in NGC 891 is characterized by a regular decrease in rotational velocity with $z$ (gradient of $\sim 15 \mathrm{~km} \mathrm{~s}^{-1} \mathrm{kpc}^{-1}$ ) (Fraternali et al. (2005)).

\section{Modelling the halo gas}

What is the origin of the gaseous haloes? Supernova explosions and stellar winds in the discs of spiral galaxies are responsible for the ejection of gas towards the halo producing a circulation called 'galactic fountain' (Shapiro \& Field (1976), Bregman (1980)). However, recent theoretical models show that the kinematics of both neutral and ionised halo gas (lag with respect to the disk gas) cannot be explained by a "pure" (ballistic) galactic fountain (Fraternali \& Binney (2006), Heald et al. (2006b)). In particular there is a need for the fountain gas to loose part of its angular momentum. Such a loss can be achieved in two ways: 1) interaction between the fountain clouds and a pre-existing hot corona or 2) interaction with accreting material.

It can be shown that the first possibility is unphysical since the hot corona has no capacity for absorbing the angular momentum of the fountain particles (Fraternali \& Binney, in preparation). The amount of gas brought up by the fountain is so high that the hot corona will be brought to corotation in a time much shorter than the dynamical time. Therefore the corona cannot be responsible for the observed rotational lag of the H I halo.

More promising models involve the interaction of the fountain clouds with cold material accreted from the IGM (Fraternali \& Binney, in preparation).

\section{References}

Boomsma, R., Oosterloo, T., Fraternali, F., van der Hulst, J.M., \& Sancisi, R., "Extra-planar Gas", Dwingeloo, ASP Conf. Series, ed. R. Braun.

Bregman, J.N., 1980, ApJ, 236, 577.

Fraternali, F. \& Binney, J.J., 2006, MNRAS, 366, 449.

Fraternali, F., van Moorsel, G., Sancisi, R., \& Oosterloo T. 2002, AJ, 123, 3124.

Fraternali, F., Oosterloo, T., Sancisi, R., \& van Moorsel, G. 2001, ApJ, 562, L47.

Fraternali, F., Oosterloo, T., Sancisi, R., \& Swaters, R.A. 2005, ASPC, 331, 239.

Heald, G.H., Rand, R.J., Benjamin, R.A., \& Bershady, M.A. 2006, ApJ, 647, 1018.

Hoopes, C.G., Walterbos, R.A.M., \& Rand, R. J. 1999, ApJ, 522, 669.

Matthews, L.D., \& Wood, K., 2003, ApJ, 593, 721.

Oosterloo, T., Fraternali, F., \& Sancisi, R. 2006, AJ, submitted.

Rossa, J., \& Dettmar, R.-J. 2003, A\&A, 406, 505.

Shapiro, P.R., \& Field, G.B., 1976, ApJ, 205, 762.

Strickland, D.K., Heckman, T.M., Colbert, E.J.M., Hoopes, C.G., \& Weaver, K.A. 2004, ApJS, 151, 193.

Swaters, R.A., Sancisi, R., \& van der Hulst, J. M. 1997, ApJ, 491, 140.

Wakker, B.P, \& van Woerden, H., 1997, ARA\&A, 35, 217.

Westmeier, T., Braun, R., \& Thilker, D. 2005, A\&A, 436, 101. 


\section{Discussion}

Gerhard Hensler: Do you see a correlation between the halo HI content and thickness or activity of the galaxy disk?

Filippo Fraternali: The sample is very limited at the moment (about 7 objects) and it is still difficult to look for correlations. There is some indication for a correlation between the total mass of the HI halo and the Star Formation Rate, but only for galaxies with low SFR.

EDWARD TAYLOR: I was wondering whether you'd made any more quantitative comparisons between the Milky Way HVCs and the substructure you have identified, including the number of clouds.

Filippo Fraternali: The structures we find in external galaxies are analogous to the HVCs and IVCs of the MW. The masses, sizes, and velocity deviations are of the same order as those of the HVCs with known distances (such as Complex A). In external galaxies we observe only the largest clouds and a comparison in number with the HVCs is difficult. 